Computing 33, 297-313(1984)

\title{
Convergence of Method of Lines Approximations to Partial Differential Equations
}

\author{
J. G. Verwer, Amsterdam, and J. M. Sanz-Serna, Valladolid, Spain
}

Received May 24, 1984

Abstract - Zusammenfassung

\begin{abstract}
Convergence of Method of Lines Approximations to Partial Differential Equations. Many existing numerical schemes for evolutionary problems in partial differential equations (PDEs) can be viewed as method of lines (MOL) schemes. This paper treats the convergence of one-step MOL schemes. Our main purpose is to set up a general framework for a convergence analysis applicable to nonlinear problems. The stability materials for this framework are taken from the field of nonlinear stiff ODEs. In this connection, important concepts are the logaritbmic matrix norm and C-stability. A nonlinear parabolic equation and the cubic Schrödinger equation are used for illustrating the ideas.
\end{abstract}

1980 Mathematics Subject Classifications: 65XX02, 65M10, 65M 20.

1982 C.R. Categories: 5.17.

Key words and phrases: Initial value problems, partial differential equations, method of lines, stiff differential equations, nonlinear problems, convergence analysis.

Konvergenz von auf der Linienmethode basierenden Approximationen partieller Differentialgleichungen. Viele numerische Verfahren für Anfangswertprobleme für partielle Differentialgleichungen kann man als Linienmethoden interpretieren. Diese Arbeit behandelt solche Verfahren vom Einschritt-Typ. Unser Ziel ist die Behandlung von Konvergenzfragen, insbesondere für nichtlineare Probleme. Unsere Hilfsmittel zum Nachweis der Stabilität entnehmen wir der stark entwickeiten Theorie für nichtlineare steife gewöhnliche Differentialgleichungen. Wichtig sind hierbei die logarithmische Matrixnorm und der C-Stabilitätsbegriff. Eine nichtlineare parabolische Gleichung und die kubische Schrödingergleichung werden verwendet, um die Ideen zu illustrieren.

\section{Introduction}

A well-known approach in the numerical solution of evolutionary problems in partial differential equations (PDEs) is the so-called method of lines (MOL). In this approach the solution process is thought of as consisting of two parts, viz., the space discretization and the time integration. In the space discretization the PDE is approximated by a system of ordinary differential equations (ODEs) by discretizing the space variable by finite differences, finite elements, spectral techniques, etc. The time $t$ is then the independent variable of the ODE system. In the second part, the time integration, this system is discretized in time to yield the final, fully discrete scheme. It is well known that many existing numerical schemes for time dependent PDEs can be viewed in this way. Concerning the time integration, we shall confine 
our discussion to the class of one-step schemes. Concerning the discretization in space, we restrict our attention to finite differences. However it should be mentioned that the treatment of finite elements or spectral methods offers no essential novelty [9].

This paper deals with the convergence of MOL schemes. Our purpose is to set up a general framework for the convergence analysis. The stability materials for this framework are borrowed from the field of nonlinear stiff ODEs. As a matter of fact, the whole analysis is centered around the semi-discrete problem. This is most convenient for the analysis and, in particular, allows for a general treatment. For example, in setting up the framework it is not necessary to distinguish between linear and nonlinear problems, although nonlinearities normally will make the hypotheses more difficult to check.

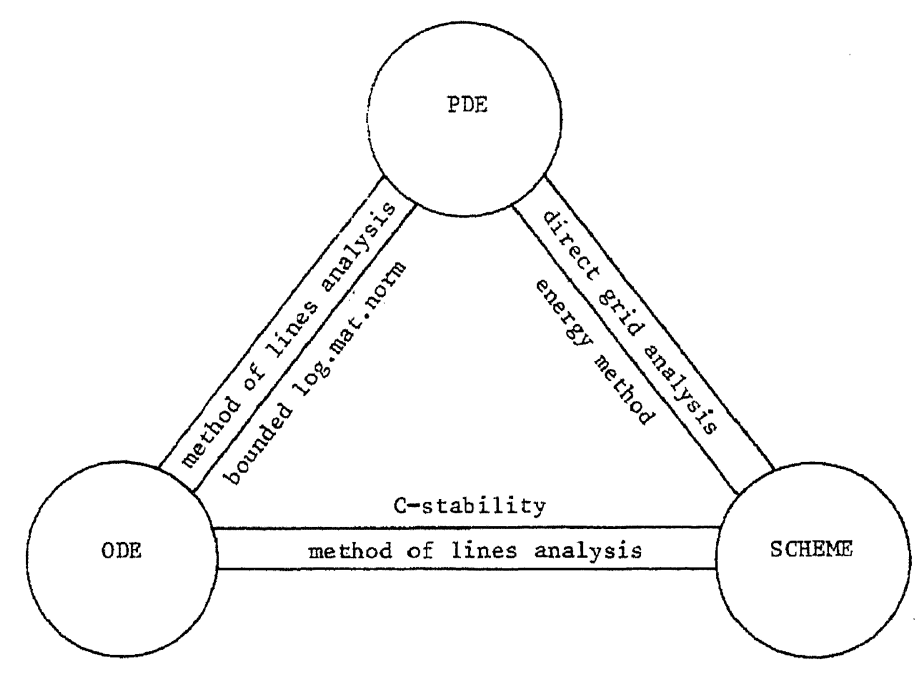

Fig. 1

The diagrammatic picture of the stability analysis shows the concepts used. In the direct grid approach, i.e., when only the PDE and the fully discrete problem are considered, one normally proves the necessary stability by using energy method arguments. In the MOL approach the necessary stability for the discretization in space can be based on the existence of a boumded logarithmic matrix norm, a concept which goes back to Dahlquist [3] (see Section 3). The concept of C-stability is employed for deciding upon the necessary stability for the time integration. $C$ stability is an abbreviation for convergence stability (cf. [11]) and is linked with stability in the Lax-Richtmyer sense and, more closely with stability in the sense of Kreiss [7]. In many applications $C$-stability can be concluded directiy from known results from the field of nonlinear stiff ODEs [4]. The existence of a bounded logarithmic matrix norm is often a prerequisite for proving $C$-stability. 
In order to give insight into the feasibility and applicability of the convergence theory we shall present a full convergence proof for approximations to a nonlinear parabolic problem (Section 5) and a nonlinear Schrödinger problem (Section 6).

This work is a sequel to the survey papers of Sanz-Serna [9] and Verwer and Dekker [11]. Part of our terminology stems from these two articles.

\section{Some Preliminaries}

We consider a real abstract Cauchy problem

$$
u_{t}=\mathscr{F}(t, u), 0<t \leq T, u(x, 0)=u^{\circ}(x),
$$

where $\mathscr{F}$ represents a partial differential operator which differentiates the unknown function $u(x, t)$ w.r. to its space variable $x$ in the space domain in $\mathbb{R}, \mathbb{R}^{2}$ or $\mathbb{R}^{3}$. $\mathscr{F}$ should not differentiate w.r. to the time variable $t$. The function $u(x, t)$ may be a vector function. Boundary conditions are supposed to be included in the definition of $\mathscr{F}$.

To the problem (2.1) we associate a real Cauchy problem for an ODE system,

$$
\dot{U}=F(t, U), 0<t \leq T, U(0)=U^{0}, F(t, \cdot): \mathbb{R}^{m} \rightarrow \mathbb{R}^{m},
$$

which is defined by a discretization of the space variable in (2.1). For the moment it is not necessary to discuss in detail how the semi-discrete, continuous time approximation (2.2) arises from (2.1). Nor is it necessary, for the time being, to be specific about the partial differential equation. The reason is that our convergence analysis is centered around the ODE system (2.2). This is most convenient for the analysis and allows for the general treatment we aim at. We merely assume that $U$ and $F$ represent the values of grid functions on a space grid covering the space domain of (2.1). Further, we let $h$ refer to the grid spacing, i.e., to the grid distances which may vary over the grid. In what follows, $\rho(h) \rightarrow 0$ means that the grid is refined arbitrary far in a suitable manner: $\rho$ stands for a distance function, e.g., the maximal distance in the grid. Note that the dimension $m$ of problem (2.2) depends on $h$.

In this paper we avoid questions concerning existence, uniqueness and smoothness of exact and numerical solutions. Hence, we suppose throughout that the two Cauchy problems at hand possess unique solutions $u(x, t)$ and $L^{\prime}(t)$. respectively. In addition, it is supposed that the true PDE solution is as smooth as the numerical analysis requires.

Let $r_{h}$ be the natural restriction operator on the space grid. We write $u_{h}(t)=r_{h} u(x, t)$. If the discretization in space is convergent, the space discretization error

$$
\eta(t)=U(t)-u_{h}(t)
$$

can be made arbitrarily small upon grid refinement. We shall discuss an error bound for $\eta$ which depends merely on the smoothness of $u_{h}$ and on the stability of the ODE system (2.2) through a logarithmic matrix norm (cf. Dahlquist [3]). This error bound exploits fully the advantage of the notion of logarithmic matrix norm which is also used later on in the paper. This error bound for $\eta$ is discussed in Section 3 . 
For the lime integration of the semi-discrete approximation $\{2.2\}$ we shall concentrate on one-step schemes. Let the implicit relation

$$
U^{n+1}=U^{n}+\tau \Phi\left[\tau, U^{n}, U^{n+1}\right], \tau=t_{n+1}-t_{n} \quad(n \geq 0)
$$

represent such a scheme. Here $t_{v}=0$ and $U^{n}$ is the approximation to $L\left(t_{n}\right)$. At this stage we let $t$ and $\rho(h)$ be independent parameters. If the scheme $(2.4)$ is a convergent integration formula, the time discretization error, or time integration error

$$
\delta\left(t_{n+1}\right)=U^{n+1}-U\left(t_{n+1}\right),
$$

will vanish as $\tau \rightarrow 0$ for any lixed grid spacing. It should be emphasized, however, that the use of a convergent ODE solver and a convergent discretization in space, not necessarily guarantees that the full discretization error

$$
\varepsilon\left(t_{n+1}\right)=U^{n+1}-u_{n}\left(t_{n+1}\right)=\delta\left(t_{n+1}\right)+\eta\left(t_{n+1}\right)
$$

will vanish for decreasing $\tau$ and $\rho(h)$. It may be necessary to impose an additional relation between $\tau$ and $\rho(h)$. A classical example is furnished by the one dimensional heat equation $u_{t}=u_{x x}$. If we discretize in space on an equidistant grid using second order finite differences and integrate in time with the forward Euler method, the well-known additional relation $\tau \leq \frac{1}{2} h^{2}$ is required [8]. The explanation of this behaviour is related to the fact that the standard bounds for $\delta\left(t_{n+1}\right)$ used in the convergence theory of ODE solvers, involve the Lipschitz constant of the system (2.2) and these constants increase with decreasing $h$. Therefore in order to achieve the convergence of $U^{n}$ to $u_{h}\left(t_{n}\right)$ as $\tau \rightarrow 0$ and $\rho(h) \rightarrow 0$, we must demand that the convergence of $L^{n}$ to $U\left(t_{n}\right)$ be uniform, in some appropriate sense, in the grid spacing. Here the recent results from the field of nonlinear stiff ODEs fit into the picture, as the Leitmotiv in those developments is the derivation of error bounds which hold uniformly with respect to the Lipschitz constant or the stiffness of the problem [4], [5]. We shall discuss these matters in Section 4.

\section{Convergence of the Discretization in Space}

Consider the two Cauchy problems $(2.1),(2.2)$. Introduce the space truncation error

$$
x(t)=F\left(t, u_{k}(t)\right)-\dot{u}_{h}(t),
$$

where $\dot{u}_{k}(t)=d u_{k}(t) d t=r_{h} u_{t}(x, t)$, i.e., the restriction of the derivative $u_{k}$ of the true PDE solution $u$ to the space grid. The defect $\alpha$ is obtained by substituting the true solution $u_{h}$ into the semi-discrete approximation. Loosely speaking, it measures how the partial differential operator $\mathscr{F}$ is approximated by the vector function $F$. The consistency of the method for a given norm means, by definition, that $\|\alpha(t)\| \rightarrow 0$ as $\rho(h) \rightarrow 0$ uniformly in $t$.

It now trivially follows that $\eta$, the discretization error in space, is a solution of the ODE system

$$
\dot{\eta}=F\left(t, u_{k}+\eta\right)-F\left(t, u_{i}\right)+\alpha(t), 0<t \leq T .
$$

Using the mean value theorem for vector functions, we can write

$$
\dot{\eta}=M(t) \eta+\alpha(t), 0<t \leq T
$$




$$
M(t)=\int_{0}^{1} F^{\prime}\left(t, u_{h}+\theta \eta\right) d \theta
$$

where $F^{\prime}(t, \cdot)$ is the Jacobian matrix of $F(t, \cdot)$.

This result shows that $\eta(t)$ depends essentially on $\alpha(t)$, which is determined completely by the smoothness of $u$ and the quality of the approximation in space, and on the stability of the ODE system (2.2). We shall give a bound for $\eta$ from which convergence of $U$ to $u_{h}$ can be derived, provided that the discretization in space is consistent. This bound leans completely upon the fundamental concept of a logarithmic matrix norm and is due to Dahlquist [3]. For details on the important role of the logarithmic matrix norm in proving stability of stiff nonlinear ODE systems the reader can also consult [4], Section 1.5 .

Introduce a norm $\|\cdot\|$ on $\mathbb{R}^{m}$. Let $\mu[\cdot]$ be its corresponding logarithmic matrix norm. Let $T_{h}(t)=\left\{\zeta: \zeta=u_{h}(t)+\theta \eta(t), 0 \leq \theta \leq 1\right\}$ and let $\mu_{\max }$ be a constant such that

$$
\mu_{\max } \geq \max _{\zeta}\left\{\mu\left[F^{\prime}(t, \zeta)\right]: \zeta \in T_{h}(t)\right\} \text { for all } t \in[0, T] \text {. }
$$

Hence for each $t$ we compute the maximal logarithmic matrix norm of $F^{\prime}$ on the line segment $T_{h}(t)$ and majorize these by $\mu_{\max }$. Then

$$
\|\eta(t)\| \leq e^{\mu_{\max }{ }^{T}}\|\eta(0)\|+\int_{0}^{2} e^{\mu_{\max }(t-\tau)}\|\alpha(\tau)\| d t .
$$

Supposing that $\|\eta(0)\|=0$, we can write

$$
\|\eta(t)\| \leq C\left(t, \mu_{\max }\right) \max _{0 \leq \tau \leq t}\|\alpha(\tau)\|, 0 \leq t \leq T,
$$

where $C\left(t, \mu_{\max }\right)=\left(e^{\mu_{\max }}-1\right) / \mu_{\max }$ depends solely on $t$ and $\mu_{\max }$. Consequently, if a constant $\mu_{\max }$ exists independent of the grid spacing, $\|\eta(t)\| \rightarrow 0$, for $\rho(h) \rightarrow 0$. Thus we can state

Theorem 3.1: Suppose that the discretization in space is consistent and that $\mu_{\max }$ exists independent of the grid spacing. Then the discretization in space is convergent.

The practical importance of this theorem lies in the fact that in many applications in the field of nonlinear, time dependent PDEs a $\mu_{\max }$ can be determined which is indeed independent of $h$ (see the Sections 5, 6 and $[9,11]$ ). Applications can also be found in the solution of stationary problems in PDEs by means of iterative methods. As it is well known many of these methods can be thought of as integrating in time a related time dependent PDE whose asymptotic solution $u_{h}(\infty)$ should give the desired stationary solution (see, e.g., [13]). Here it is required that $\mu_{\max }<0$.

The inequality (3.5) is in fact nothing else than a stability inequality for the Cauchy problem (2.2). To see this, let $U, \tilde{U}$ be two solutions belonging to two different initial values $U^{0}$ and $\tilde{U}^{0}$, respectively. Let $\mu_{\max }$ now satisfy

$$
\mu_{\max } \geq \max _{\zeta}\left\{\mu\left[F^{\prime}(t, \zeta)\right]: \zeta=\theta U(t)+(1-\theta) \tilde{U}(t), 0 \leq \theta \leq 1\right\}, 0 \leq t \leq T .
$$

Then (cf. [3]),

$$
\|\bar{U}(t)-U(t)\| \leq e^{\mu_{\max }{ }^{2}}\left\|\hat{U}_{0}-U_{0}\right\|, 0 \leq t \leq T
$$


The inequality (3.5) is equivalent to this stability inequality which can be refined somewhat by taking $\mu_{\max }$ time dependent. The growth factor $\exp \left(\mu_{\max } i\right)$ is then replaced by

$$
\exp \left(\int_{0}^{t} \mu_{\max }(\tau) d \tau\right)
$$

Observe that the inequality $\left(3.5^{\prime}\right)$ trivially implies uniqueness of solutions for system (2.2) for any grid spacing.

Remark 3.1: For the standard $l^{p}$ norms the expression for $\mu$ is known. Let $A=\left(a_{i j}\right)$ be a real $m \times m$ matrix. For the norms $\|\cdot\|_{1}$ and $\|\cdot\|_{\infty}$ on $\mathbb{R}^{m}$,

$$
\mu_{1}[A]=\max _{j}\left(\alpha_{i j}+\sum_{i \neq j}\left|a_{i j}\right|\right), \quad \mu_{\alpha}[A]=\max _{i}\left(a_{i i}+\sum_{j \neq i}\left|a_{i j}\right|\right) .
$$

For inner product norms, $\|\zeta\|=\langle\zeta, \zeta\rangle^{1 / 2}$

$$
\mu[A]=\max _{\zeta \neq 0}\langle A \zeta, \zeta\rangle i\|\zeta\|^{2} .
$$

Hence, for the spectral norm, $\mu_{2}[A]$ is the maximal eigenvalue of the symmetric part $\left(A+A^{T}\right) / 2$ of $A$.

Remark 3.2: It follows from (3.9) that $\mu[A] \leq v$ if $v$ represents a one-sided Lipschitz constant of $A$, i.e., $\langle A \zeta, \zeta\rangle \leq v\|\zeta\|^{2}, \forall \zeta \in \mathbb{R}^{m}$. Loosely speaking, the logarithmic matrix norm might be considered as a generalization of the one-sided Lipschitz constant for norms other than inner product norms. When dealing with inner product norms it is sometimes more convenient to use the one-sided Lipschitz condition for $F$,

$$
\begin{gathered}
\left\langle F\left(t, \zeta_{1}\right)-F\left(t, \zeta_{2}\right), \zeta_{1}-\zeta_{2}\right\rangle \leq v\left\|\zeta_{1}-\zeta_{2}\right\|^{2}, \\
\forall \zeta_{1}, \zeta_{2} \in T_{h}(t), \forall t \in[0, T],
\end{gathered}
$$

rather than computing $F^{\prime}$ and using (3.9). Any constant $v$ satisfying (3.4"), a onesided Lipschitz constant for $F$, may be used for $\mu_{\max }$ in the inequalities (3.4), (3.4').

Remark 3.3: Obviously, the accuracy of $U$ will depend largely on the magnitude of the truncation error $\alpha$. For finite times, i.e., $T<\infty$, the bound (3.6) shows that $\eta \eta(t) \|$ has the same rate of convergence as $\|\alpha(t)\|$, provided that $\mu_{\max }$ exists independent of $h$. If $\mu_{\max }<0$, this bound also applies to Cauchy problems on infinite time intervals. For dissipative problems it is often possible to prove the existence of a negative $\mu_{\max }$. If $\mu_{\max }=0$, (3.6) allows a linear growth of the space error in time. If $\mu_{\max }>0$, it allows an exponential growth of this error in time and will mostly be rather pessimistic on long time intervals.

\section{Convergence of the Full Discretization}

Consider the integration method (2.4) for the Cauchy problem (2.2). in this section we shall study the full convergence of this method as $\tau \rightarrow 0$ and $\rho(h) \rightarrow 0$. For ease of presentation we restrict ourselves to constant stepsizes $\tau$, i.e., in the limit process we take $t_{n+1} \in(0, T]$ fixed and suppose that $\tau \rightarrow 0, n \rightarrow \infty$ in such a way that 
$(n+1) \tau=t_{n+1}$. The restriction to constant stepsizes is not essential for our results and, as it is well known, can be removed.

Let us introduce the full truncation error

$$
\begin{gathered}
\beta\left(t_{n+1}\right)=\hat{U}^{n+1}-u_{h}\left(t_{n+1}\right), \\
\hat{U}^{n+1}=u_{h}\left(t_{n}\right)+\tau \Phi\left[\tau, u_{h}\left(t_{n}\right), \hat{U}^{n+1}\right] .
\end{gathered}
$$

Observe that $\beta$ is defined with respect to the true PDE solution and not for the true ODE solution $U$. If we had used $U$ the time truncation error

$$
\gamma\left(t_{n+1}\right)=\bar{U}^{n+1}-U\left(t_{n+1}\right), \bar{U}^{n+1}=U\left(t_{n}\right)+\tau \Phi\left[\tau, U\left(t_{n}\right), \bar{U}^{n+1}\right]
$$

would have been obtained which is considered normally in numerical ODE theory. In the setting of PDEs the error (4.1) is to be preferred for the convergence analysis for reasons which will be discussed in Section 7. Note that, in a sense, the full truncation error $\beta$ contains the space truncation error $\alpha$, given by (3.1), as the increment operator $\Phi$ of the integration formula depends on the ODE operator $F$.

Let us express the full discretization error $\varepsilon\left(t_{n+1}\right)$, given by (2.6), as

$$
\varepsilon\left(t_{n+1}\right)=U^{n+1}-\hat{U}^{n+1}+\beta\left(t_{n+1}\right),
$$

and suppose that for a positive $\kappa \in \mathbb{R}$ and a norm on $\mathbb{R}^{m}$,

$$
\left\|\hat{U}^{n+1}-U^{n+1}\right\| \leq \kappa\left\|\hat{U}^{n}-U^{n}\right\|, \hat{U}^{n}=u_{h}\left(t_{n}\right) .
$$

Then $\left\|\varepsilon\left(t_{n+1}\right)\right\| \leq \kappa\left\|\varepsilon\left(t_{n}\right)\right\|+\chi$, where $\chi$ is an upper bound for $\beta$. The error $\varepsilon\left(t_{n+1}\right)$, i.e., the error after $n+1$ time steps, then is easily shown to satisfy

$$
\left\|\varepsilon\left(t_{n+1}\right)\right\| \leq \kappa^{n+1}\left\|\varepsilon\left(t_{0}\right)\right\|+\chi \frac{1-\kappa^{n+1}}{1-\kappa}, \kappa \neq 1 .
$$

This standard inequality is the starting point for the full convergence analysis which is based on the concept of $C$-stability. In the definition below a second numerical solution $\tilde{U}^{n+1}$ is considered, i.e., $\tilde{U}^{n+1}=\tilde{U}^{n}+\tau \Phi\left[\tau, \widetilde{U}^{n}, \widetilde{U}^{n+1}\right]$.

Definition 4.1: Let $|\cdot|$ be a norm on $\mathbb{R}^{m}$. The integration method is called $C$-stable for the Cauchy problem (2.2), with respect to this norm, if a positive real number $\tau_{0}=\tau_{0}(h)$ and a real constant $C_{0}$, independent of $\tau$ and $h$ exist, such that for each $\tau \in\left(0, \tau_{0}\right]$ and each $U^{n}, \tilde{U}^{n} \in \mathbb{R}^{m}$

$$
\left\|\tilde{U}^{n+1}-U^{n+1}\right\| \leq\left(1+C_{0} \tau\right)\left\|\tilde{U}^{n}-U^{n}\right\| .
$$

Remark 4.1: Note that for $\tilde{U}^{n}, \tilde{U}^{n+1}$ one may substitute $u_{h}\left(t_{n}\right)$ and $\hat{U}^{n+1}$ (cf. (4.3)).

$C$-stability is an abbreviation for "convergence stability" and is linked with stability in the Lax-Richtmyer sense [8] and, more closely with stability in the sense of Kreiss [7] (sometimes referred to as strong stability [8]). If $C_{0} \leq 0$ and we think of $U^{n}$, as being a numerical solution, and of $\tilde{U}^{n}$ as being a perturbation of $U^{n}$, then (4.5) shows that the perturbation will not increase in time. The bound (4.5) then provides the 
definition of contractivity, also called "computing stability" [11], a concept which plays a major role in recent developments in ODEs [4]. If $C_{0}>0$, we allow an increase in the difference $\tilde{U}^{n}-U^{n}$. In this case $C$-stability is mainly useful in the convergence analysis and not as a concept of "computing stability". Finally note that the essence of $C$-stability in the context of PDEs, is that $C_{0}$ is independent of the grid spacing.

Let us now suppose that for a given Cauchy problem (2.2) the integration method is $C$-stable. We then may substitute $k=1+C_{0} \tau$ into the bound (4.4). An easy derivation yields the familiar expressions

$$
\left\|\varepsilon\left(t_{n+1}\right)\right\| \leq \begin{cases}\tau^{-1} \chi\left|C_{0}\right|^{-1}, & C_{0}<0, \\ \tau^{-1} \chi t_{n+1}, & C_{0}=0, \\ \tau^{-1} \chi C_{0}^{-1}\left(e^{C_{0} t_{n+1}}-1\right), & C_{0}>0,\end{cases}
$$

where, for convenience, $\left\|\varepsilon\left(t_{0}\right)\right\|$ is taken to be zero. By the hypotheses of $C$-stability, we can conclude that this error bound is valid uniformly in the grid spacing under the stepsize restriction $\tau \in\left(0, \tau_{0}(h)\right]$. Note that for $C_{0}<0$ (strict contractivity) the bound is useful for infinite time intervals.

Finally we suppose that the full discretization is consistent, i.e.,

$$
\left\|\tau^{-1} \beta\left(t_{n+1}\right)\right\| \rightarrow 0 \text { as } \tau, \rho(h) \rightarrow 0 \text {, }
$$

uniformly in $n$. It is then evident that we can state

Theorem 4.1: Suppose that the full discretization is consistent and the inteysation method C-stable. Then the full discretization is convergent.

At this place we want to emphasize that the stepsize restriction for $C$-stability, i.e., $\tau \in\left(0, \tau_{0}(h)\right]$, may lead to additional conditions on the refinement of the time- and space grid. It is well known that for explicit integration formulas $\tau_{0}(h) \rightarrow 0$ as $\rho(h) \rightarrow 0$.

Remark 4.2: The C-stability theory of implicit Runge-Kutta methods for stiff ODEs, where the statement "independent of the grid spacing" is to be replaced by "independent of the stiffness", has already been developed to a considerable extent [4]. For example, for constant coefficient linear systems dissipative in inner product spaces the celebrated property of $A$-stability implies $C$-stability where $C_{0}=0$. Likewise, the property of $B$-stability [2] for nonlinear dissipative problems is a $C$-stability property where again $C_{0}=0$. A general result for non-linear problems satisfying the one-sided Lipschitz condition (3.4") can be found in [4], Theorem 7.4.2. Because of the intimate relationship with semi-discrete PDEs many results from the field of nonlinear stiff ODEs apply to PDEs in a straightforward manner. For integration methods not belonging to the class of implicit Runge-Kutta methods the hypotheses of $C$-stability must be verified separately. By way of illustration we shall devote the Sections 5 and 6 to two examples. 


\section{A Nonlinear Parabolic Equation}

We consider a nonlinear, one space dimensional, scalar parabolic initial-boundary value problem of the type (cf. [12])

$$
\begin{gathered}
u_{t}=f\left(t, x, u, \frac{\partial}{\partial x}\left(p(t, x) \frac{\partial u}{\partial x}\right)\right), 0<t \leq T, x \in \Omega=(0,1), \\
u(0, t)=b_{0}(t), u(1, t)=b_{1}(t), 0<t \leq T \\
u(x, 0)=u^{0}(x), 0 \leq x \leq 1
\end{gathered}
$$

where the functions $p(t, x)$ and $f(t, x, a, b)$ satisfy the familiar conditions

$$
\begin{gathered}
p(t, x) \geq p_{0}>0,0<t \leq T, x \in \Omega, \\
\partial f(t, x, a, b) / \partial b \geq f_{0}>0,0<t \leq T, x \in \Omega, a, b \in \mathbb{R} .
\end{gathered}
$$

Here $p_{0}$ and $f_{0}$ are constants. In addition, we suppose that there exist real numbers $f_{-1}, f_{1}$ such that

$$
f_{-1} \leq \partial f(t, x, a, b) / \partial a \leq f_{1}, 0<t \leq T, x \in \Omega, a, b \in \mathbb{R} .
$$

\subsection{Discretization in Space}

We space discretize the problem on a nonequidistant finite difference grid. Define

$$
\Omega_{h}=\left\{x_{j}: x_{j}=x_{j-1}+h_{j}, j=1(1) m ; x_{0}=0, x_{m+1}=1\right\} .
$$

Apply 3-point finite differences for the discretization of $\left(p(t, x) u_{x}\right)_{x}$. Let $U_{j}$ and $F_{j}$ be the $j$-th component of the grid functions $U$ and $F$, respectively. Then, for $j=1(1) m$,

$F_{j}(t, U)=f\left(t, x_{j}, U_{j},\left(\frac{2}{x_{j+1}-x_{j-1}}\right)\left(s_{j}^{-} U_{j-1}-\left(s_{j}^{-}+s_{j}^{+}\right) U_{j}+s_{j}^{+} U_{j+1}\right)\right)$,

where

and

$$
s_{j}^{ \pm}=p\left(t, \frac{1}{2}\left(x_{j}+x_{j \pm 1}\right)\right) /\left|x_{j}-x_{j \pm 1}\right|
$$

$$
U_{0}(t)=b_{0}(t), U_{m+1}(t)=b_{1}(t)
$$

Let us prove convergence of $U$ to $u_{h}$ in $l^{\infty}$, i.e., in the maximum norm $\|U\|_{\infty}=\max _{j}\left|U_{j}\right|$, along the lines of Section 3. The logarithmic matrix norm $\mu_{\infty}$ is given by

$$
\mu_{\infty}[A]=\max _{i}\left(a_{i i}+\sum_{j \neq i}\left|a_{i j}\right|\right),
$$

for any real $m \times m$ matrix $A=\left(a_{i j}\right)$. Let $\bar{U}$ be a point on the line segment $T_{h}(t)$ which appears in the inequality (3.4) for the logarithmic norm. The Jacobian matrix $F^{\prime}(t, \bar{U})$ of the vector function $F(t, \cdot)$ defined by $(5.4)$ is of the form

$$
F^{\prime}(t, \bar{U})=D_{1}+D_{2} D_{3} S,
$$

where $D_{1}, D_{2}, D_{3}$ are $m \times m$ diagonal matrices and $S$ is a symmetric tridiagonal $m \times m$ matrix. The entries of $D_{1}$ are the derivatives of $f$ to the third argument. All entries are

21 Computing 33/3-4 
less than or equal to the constant $f_{1}$. The entries of $D_{2}$ are the derivarives of $f$ w.r. to the fourth argument and, by assumption, $D_{2}$ is positive definite. $D_{3}$ contains all numbers $2 /\left(x_{j+1}-x_{j-1}\right)$, hence is also positive definite. Finally, the entries in the $j$-th row of $S$ are just the numbers $s_{j}^{-},-\left(s_{j}^{-}+s_{j}^{+}\right), s_{j}^{+}$. It follows that $S$ is diagonally dominant with negative diagonal entries.

Now we are ready to compute $\mu_{\infty}\left[F^{\prime}(t, \bar{U})\right]$. First, we conclude that $\mu_{x_{1}}[\mathrm{~S}]=0$. Then, using the positive definiteness of $D_{2}$ and $D_{3}$, we find $\mu_{\infty}\left[\mathrm{D}_{2} \mathrm{D}_{3} \mathrm{~S}\right]=0$, too. It follows that

$$
\mu_{\infty}\left[F^{\prime}(t, \bar{U})\right] \leq f_{1}, 0 \leq t \leq T .
$$

Clearly, this inequality is valid for any $\bar{U} \in T_{h}(t), 0 \leq t \leq T$ (it is even valid for $\bar{U}$ arbitrary in $\mathbb{R}^{m}$ ). Hence we have found a bound $\mu_{\max }=f_{1}$ satisfying (3.4) for any $h$, and $\mu_{\max }$ is independent of $h$. There remains to verify the consistency of the space discretization. As it is well known, any component $\alpha_{j}$ of the truncation error (3.1) satisfies

$$
\alpha_{j}=O(\rho(h)) \quad \text { as } \quad \rho(h) \rightarrow 0, \rho(h)=\max _{j}\left(h_{j}\right),
$$

provided that $\max \left(h_{j}\right) / \min \left(h_{j}\right)$ remains bounded. Consequently, the discretization is consistent of order one. We have $\|\alpha(t)\|_{\infty}=O(\rho(h)), 0 \leq t \leq T$. The convergence in $l^{\infty}$ follows from Theorem 3.1 .

\subsection{Discretization in Space and Time}

Along the lines of Section 4 we shall prove $l^{\infty}$ convergence of the two fully discrete schemes which are obtained by applying the implicit and explicit Euler rules

$$
\begin{gathered}
U^{n+1}=U^{n}+\tau F\left(t_{n+1}, U^{n+1}\right), \\
U^{n+1}=U^{n}+\tau F\left(t_{n}, U^{n}\right),
\end{gathered}
$$

to the semi-discrete continuous time system (5.4). We first consider the implicit scheme. Its $C$-stability can be concluded directly from the information available on the semi-discrete approximation using known results from the field of stiff ODEs. Let $\widetilde{U}^{n}, \widetilde{U}^{n+1}$ be a second implicit Euler solution. Using (5.7) and Theorem 2.4.1 of [4] we find that, if $\tau f_{1}<1$, then

$$
\left\|\tilde{U}^{n+1}-U^{n+1}\right\|_{\infty} \leq \frac{1}{1-\tau f_{1}}\left\|\tilde{U}^{n}-U^{n}\right\|_{\infty} .
$$

This means $C$-stability under the stepsize restriction $\tau f_{1}<1$. Note that the amplification factor and the restriction on $\tau$ are both independent of the grid spacing and are in fact valid in the whole numerical solution space due to the introduction of the constant $f_{1}$ in (5.3).

We next examine the full truncation error (4.1) of the implicit scheme. According to the definition (3.1) of the space truncation error the true PDE solution satisfies

$$
u_{h}\left(t_{n+1}\right)=\left[u_{h}\left(t_{n+1}\right)-\tau \propto\left(t_{n+1}\right)-\tau \dot{u}_{h}\left(t_{n+1}\right)\right]+\tau F\left(t_{n+1}, u_{h}\left(t_{n+1}\right)\right),
$$


while the local implicit Euler solution $\hat{U}^{n+1}$ computed from $u_{h}\left(t_{n}\right)$ satisfies

$$
\hat{U}^{n+1}=u_{h}\left(t_{n}\right)+\tau F\left(t_{n+1}, \hat{U}^{n+1}\right) \text {. }
$$

By interpreting the relation $(5.12)$ as an implicit Euler step, with the bracketed term playing the role of $U^{n}$, application of the $C$-stability inequality (5.11) to the equations (5.12), (5.13) yields directly

$$
\begin{gathered}
\left\|\beta\left(t_{n+1}\right)\right\|_{\infty} \leq \frac{1}{1-\tau f_{1}}\left\|u_{h}\left(t_{n+1}\right)-u_{h}\left(t_{n}\right)-\tau \dot{u}_{h}\left(t_{n+1}\right)-\tau \alpha\left(t_{n+1}\right)\right\|_{\infty} \\
=\frac{1}{1-\tau f_{1}}\left\|O\left(\tau^{2}\right)+\tau \alpha\left(t_{n+1}\right)\right\|_{\infty},
\end{gathered}
$$

for all $\tau f_{1}<1$. This proves the consistency of the full discretization and according to Theorem 4.1, convergence for $\tau, \rho(h) \rightarrow 0$ without any further restriction on $\tau$ and $\rho(h)$. We emphasize that the second derivative with respect to $t$ of the true PDE solution $u_{h}$ appears in the bound.

We now proceed with the explicit Euler scheme. The truncation error $\beta\left(t_{n+1}\right)$ is given by

$$
\begin{aligned}
\beta\left(t_{n+1}\right) & =u_{h}\left(t_{n}\right)+\tau F\left(t_{n}, u_{h}\left(t_{n}\right)\right)-u_{h}\left(t_{n+1}\right) \\
& =u_{h}\left(t_{n}\right)+\tau \dot{u}_{h}\left(t_{n}\right)-u_{h}\left(t_{n+1}\right)+\tau \alpha\left(t_{n}\right) \\
& =O\left(\tau^{2}\right)+\tau \alpha\left(t_{n}\right),
\end{aligned}
$$

showing consistency. Next the $C$-stability. Let $\widetilde{U}^{n}, \tilde{U}^{n+1}$ be a second explicit Euler solution. Then, using the mean value theorem,

$$
\begin{gathered}
\tilde{U}^{n+1}-U^{n+1}=\left(I+\tau M\left(t_{n}\right)\right)\left(\tilde{U}^{n}-U^{n}\right), \\
M\left(t_{n}\right)=\int_{0}^{1} F^{\prime}\left(t_{n}, \theta \tilde{U}^{n}+(1-\theta) U^{n}\right) d \theta .
\end{gathered}
$$

For obtaining a $C$-stability result, we must compute the maximum of $\left\|I+\tau F^{\prime}\left(t_{n}, \zeta\right)\right\|_{\infty}$ on the line segment $\zeta=\theta \widetilde{U}^{n}+(1-\theta) U^{n}, \theta \in[0,1]$. Consider the Jacobian matrix (5.6). Let $D_{i j}$ be the $j$-th entry of the diagonal matrix $D_{i}$ for $i=1,2,3$. Then

$$
\begin{aligned}
\max _{\zeta}\left\|I+\tau F^{\prime}\left(t_{n}, \zeta\right)\right\|_{\infty}= & \max _{\zeta} \max _{j}\left\{\left|1+\tau D_{1 j}-\tau D_{2 j} D_{3 j}\left(s_{j}^{-}+s_{j}^{+}\right)\right|\right. \\
& \left.+\tau D_{2 j} D_{3 j}\left(s_{j}^{-}+s_{j}^{+}\right)\right\} \\
= & 1+\tau \max _{\zeta} \max _{j} D_{1 j} \leq 1+\tau f_{1},
\end{aligned}
$$

if, for all $\zeta$ and all $j$,

$$
\tau\left(D_{2 j} D_{3 j}\left(s_{j}^{-}+s_{j}^{+}\right)-D_{1 j}\right) \leq 1 .
$$

To appraise these inequalities, suppose first that the parabolic equation is $u_{t}=u_{x x}$ and that $\Omega_{h}$ is equidistant (see (5.4)). Then, for all $j, D_{1 j}=0, D_{2 j}=1, D_{3 j}=h^{-1}$ and $s_{j}^{-}=s_{j}^{+}=h^{-1}$, so that the inequality reduces to the well known stability result $\tau \leq \frac{1}{2} h^{2}$. Consequently, in case of $u_{t}=u_{x x}$ and $\Omega_{h}$ equidistant, explicit Euler is $C$ stable in $l^{\infty}$ under the stepsize restriction $\tau \leq \frac{1}{2} h^{2}$.

$21^{*}$ 
Let us return to the general case. Inequality (5.18) yields a bound for $\tau$ (the bound $\tau_{0}$ in Definition 4.1). However, this bound still depends on the numerical solution $U^{n}$ through the diagonal matrix entries $D_{1 j}$ and $D_{2 j}$. The dependence is removed by using the hypotheses $(5.2 \mathrm{~b}),(5.3)$. If $\tau$ satisfies

$$
\tau\left(f_{0} D_{3 j}\left(s_{j}^{-}+s_{j}^{+}\right)-f_{-1}\right) \leq 1,
$$

we thus arrive at (for any pair of numerical solutions)

$$
\left\|\tilde{U}^{n+1}-U^{n+1}\right\|_{\infty} \leq\left(1+\tau f_{1}\right)\left\|\tilde{U}^{n}-U^{n}\right\|_{\infty} .
$$

This proves $C$-stability in $l^{\infty}$ of explicit Euler for the semi-discrete system (5.4) under the stepsize restriction

$$
0<\tau \leq\left(f_{0} D_{3 j}\left(s_{j}^{-}+s_{j}^{+}\right)-f_{-1}\right)^{-1}
$$

\section{The Cubic Schrödinger Equation}

From [10] we quote the following initial-boundary value problem for the cubic Schrödinger equation (here $u=[v, w]^{T}$ ),

$$
\begin{gathered}
v_{t}+w_{x x}+\left(v^{2}+w^{2}\right) w=0,0<t \leq T, x \in \Omega=\left(x_{L}, x_{R}\right), \\
w_{t}-v_{x x}-\left(v^{2}+w^{2}\right) v=0,0<t \leq T, x \in \Omega=\left(x_{L}, x_{R}\right), \\
v_{x}(x, t)=w_{x}(x, t)=0, x=x_{L}, x_{R} \text { and } 0<t \leq T, \\
v(x, 0)=v^{0}(x), w(x, 0)=w^{0}(x), x_{L} \leq x \leq x_{R} .
\end{gathered}
$$

\subsection{Discretization in Space}

Let us space discretize (6.1) on the equidistant grid

$$
\begin{aligned}
\Omega_{h}=\left\{x_{1}=x_{L}, x_{j}=x_{j-1}+h(2 \leq j \leq N-1),\right. \\
\left.x_{N}=x_{R} ; h=\left(x_{R}-x_{L}\right) /(N-1)\right\}
\end{aligned}
$$

for a given integer $N$. Suppose that standard second order finite differences are used for $u_{x}$ and $u_{x x}$. Let $V_{j}(t)$ and $W_{j}(t)$ be the resulting approximation for $v\left(x_{j}, t\right)$ and $w\left(x_{j}, t\right), 1 \leq j \leq N$. The semi-discrete, continuous time approximation to $(6.1)$ is then given by, $j=1(1) N$,

where

$$
\begin{gathered}
\dot{V}_{j}+h^{-2}\left(W_{j+1}-2 W_{j}+W_{j-1}\right)+\left(V_{j}^{2}+W_{j}^{2}\right) W_{j}=0, \\
\dot{W}_{j}-h^{-2}\left(V_{j+1}-2 V_{j}+V_{j-1}\right)-\left(V_{j}^{2}+W_{j}^{2}\right) V_{j}=0,
\end{gathered}
$$

$$
V_{0}=V_{2}, W_{0}=W_{2} \text { and } V_{N+1}=V_{N-1}, W_{N+1}=W_{N-1}
$$

in accordance with the boundary conditions.

Let $U_{j}=\left[V_{j}, W_{j}\right]^{T}, U=\left[U_{1}^{T}, \ldots, U_{N}^{T}\right]^{T}$. The system can then be rewritten as

$$
\dot{U}=F(U)=(S+B(U)) U,
$$


where $S$ is the block tridiagonal matrix

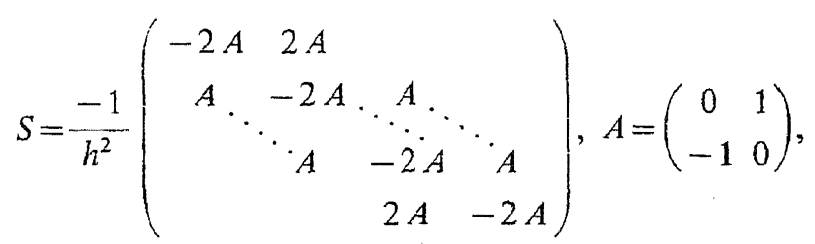

and $B(U)$ is block diagonal, i.e.,

$$
\begin{gathered}
B(U)=-\operatorname{diag}\left(B_{1}\left(U_{1}\right), \ldots, B_{N}\left(U_{N}\right)\right), \\
B_{j}\left(U_{j}\right)=\left(\begin{array}{cc}
0 & V_{j}^{2}+W_{j}^{2} \\
-V_{j}^{2}-W_{j}^{2} & 0
\end{array}\right) .
\end{gathered}
$$

This form is more convenient for our convergence analysis.

We shall prove convergence of $U(t)$ to $u_{h}(t)$ in the norm associated to the inner product

$$
\langle U, \tilde{U}\rangle_{2}=h \sum_{j=1}^{N} " U_{j}^{T} \widetilde{U}_{j}, U, \tilde{U} \in \mathbb{R}^{m}, m=2 N .
$$

The double quote means that the first and last element in the sum are multiplied by $\frac{1}{2}$. Observe that system (6.5) is conservative for this norm, i.e., $\|U(t)\|_{2}$ is constant in time [10]. For the $l^{2}$ norm, the logarithmic norm $\mu_{2}$ is

$$
\mu_{2}[A]=\max _{\zeta \neq 0} \frac{\langle A \zeta, \zeta\rangle_{2}}{\|\zeta\|_{2}^{2}}, A \text { a real } m \times m \text { matrix }
$$

Let $\bar{U}$ be a point on the line segment $T_{h}(t)$. The Jacobian matrix $F^{\prime}(\bar{U})$ can be written as

$$
F^{\prime}(\bar{U})=S+\operatorname{diag}\left(D_{j}\right), D_{j}=\left(\begin{array}{cc}
-2 \bar{V}_{j} \bar{W}_{j} & -\bar{V}_{j}^{2}-3 \bar{W}_{j}^{2} \\
3 \bar{V}_{j}^{2}+\bar{W}_{j}^{2} & 2 \bar{V}_{j} \bar{W}_{j}
\end{array}\right) .
$$

Because $S$ is skew-symmetric, $\langle S \zeta, \zeta\rangle=0, \forall \zeta$, so that

$$
\mu_{2}\left[F^{\prime}(\bar{U})\right]=\mu_{2}\left[\operatorname{diag}\left(D_{j}\right)\right] \leq \max _{j}\left(\bar{V}_{j}^{2}+\bar{W}_{j}^{2}\right) .
$$

It follows that (3.4) can be satisfied for a $\mu_{\max }$ independent of $h$ if $\bar{V}_{j}^{2}+\bar{W}_{j}^{2}$ remains bounded as $h \rightarrow 0$ for $\forall t \in[0, T]$.

In order to remove this additional hypothesis on boundedness of the semi-discrete solution we now resort to a standard argument which was also used in [10]. Consider, instead of the problem (6.1),

$$
\begin{gathered}
v_{t}+w_{x x}+a\left(v^{2}+w^{2}\right) w=0,0<t \leq T, x \in \Omega=\left(x_{L}, x_{R}\right), \\
w_{t}-v_{x x}-a\left(v^{2}+w^{2}\right) v=0,0<t \leq T, x \in \Omega=\left(x_{L}, x_{R}\right), \\
v_{x}(x, t)=w_{x}(x, t)=0, x=x_{L}, x_{R} \text { and } t>0, \\
v(x, 0)=v^{0}(x)=w^{0}(x), x_{L} \leq x \leq x_{R},
\end{gathered}
$$

where $a(z): \mathbb{R} \rightarrow \mathbb{R}$ is a smooth function such that (i) $a(z)=z$ in a neighbourhood of 
the exact solution $\left\{u(x, t), x_{L} \leq x \leq x_{R}, 0 \leq t \leq T\right\}$ of the problem (6.1) (ii) $\left|a^{\prime}(z)\right| z$ is bounded. Obviously, such a function can be constructed.

Lemma 6.1: The initial-boundary value problem (6.12) has a unique solution which is just the solution $u=[v, w]^{T}$ of the problem (6.1).

Proof: It was assumed that the problem (6.1) has a unique solution $u=[v, w]^{T}$. Clearly, this solution $u$ also satisfies (6.12) because due to hypothesis (i) on the function $a(z), a\left(v^{2}+w^{2}\right)=v^{2}+w^{2}$. To prove that this solution is unique, we suppose that a second solution exists, say $\hat{u}=[\hat{v}, \hat{w}]^{T}$, satisfying the homogeneous Neumann conditions and the initial condition $\hat{u}(x, 0)=\left[v^{0}(x), w^{0}(x)\right]^{T}$. Now consider the energy functional

$$
E(t)=\int_{x_{L}}^{x_{R}}\left[(v-\hat{v})^{2}+(w-\hat{w})^{2}\right] d x, 0 \leq t \leq T,
$$

which satisfies $E(0)=0$. It can be shown that $E(t)$ satisfies a differential inequality $\dot{E}(t) \leq C E(t), 0<t \leq T, C$ being a positive constant. Consequently, $E(t)=0$ for all $t \in[0, T]$. Thus there is at most one solution.

Let $\dot{U}=F_{a}(U)$ be the semi-discrete approximation to (6.12). A calculation similar to that for the original system (6.5) reveals that the logarithmic norm $\mu_{2}\left[F_{a}^{\prime}(\bar{U})\right]$ satisfies

$$
\mu_{2}\left[F_{a}^{\prime}(\bar{U})\right] \leq \max _{j}\left|a^{\prime}\left(z_{j}\right)\right| z_{j}, z_{j}=\bar{V}_{j}^{2}+\bar{W}_{j}^{2} .
$$

By hypothesis (ii) on $a(z)$, for $\dot{U}=F_{a}(U)$ a $\mu_{\max }$ exists independent of $h$. Because $\dot{U}=F_{a}(U)$ is a consistent approximation (of order two) to (6.12), its solution $U$ thus converges in $l^{2}$ to the solution $u_{h}$ of (6.12) as $h \rightarrow 0$. This implies also convergence in $l^{\infty}\left(\|\cdot\|_{\infty} \leq h^{-1 / 2}\|\cdot\|_{2}\right)$ so that from Lemma 6.1 and hypothesis (i) on $a(z)$ it now. follows that for $h$ small enough the solutions of both semi-discrete systems coincide. This observation completes the proof of the boundedness of the term $\bar{V}_{j}^{2}+\bar{W}_{j}^{2}$ in the inequality (6.11).

Summarizing, according to Theorem 3.1 we have proved that the ODE system (6.5) is a second order convergent approximation in $l^{2}$ to the cubic Schrödinger problem (6.1) (the proof of second order consistency is trivial).

\subsection{Discretization in Space and Time}

Along the lines of Section 4 we shall prove $l^{2}$ convergence of the fully discrete scheme defined by the implicit midpoint rule

$$
U^{n+1}=U^{n}+\tau F\left(\frac{1}{2} U^{n}+\frac{1}{2} U^{n+1}\right) \text {. }
$$

This is an obvious scheme for conservative problems such as $(6.5)$ as it is conservative, too, i.e., $\left\|U^{n}\right\|_{2}=U(0) \|_{2}(n \geq 0)$. A particular predictor corrector implementation of (6.15) which exploits the pseudo-linear form of the Schrödinger equation was studied in [6].

The proof of $C$-stability can be stated again from known results $[1,2,4,5,12]$ from the field of nonlinear stiff ODEs. Let $\tilde{U}^{n}, \tilde{U}^{n+1}$ be a second implicit mid-point 
solution. Then, using (6.10), (6.11) and the inequality (4.5) from [12], it follows that

$$
\left\|\tilde{U}^{n+1}-U^{n+1}\right\|_{2} \leq\left(\frac{2+\tau \nu}{2-\tau \nu}\right)\left\|\tilde{U}^{n}-U^{n}\right\|_{2}, \quad 0 \leq \tau \nu<2,
$$

for any one-sided Lipschitz constant $v>\max _{j}\left(\bar{V}_{j}^{2}+\bar{W}_{j}^{2}\right), \bar{U}$ now being a point on the line segment connecting $\frac{1}{2}\left(U^{n}+U^{n+1}\right)$ and $\frac{1}{2}\left(\tilde{U}^{n}+\tilde{U}^{n+1}\right)$. Consequently, the implicit midpoint rule is $C$-stable for the Schrödinger problem (6.5) if componentwise the implicit midpoint solution remains bounded as $h \rightarrow 0$. Let us assume, for the moment, that this is true and let us proceed with the proof of consistency.

For this purpose it is convenient to introduce the defect (sometimes called truncation error, too)

$$
d_{1}\left(t_{n+1}\right)=u_{h}\left(t_{n}\right)+\tau F\left(\frac{1}{2} u_{h}\left(t_{n}\right)+\frac{1}{2} u_{h}\left(t_{n+1}\right)\right)-u_{h}\left(t_{n+1}\right) .
$$

Note that $d_{1}$ depends solely on the true PDE solution. The full truncation error $\beta$ is given by

We write

$$
\begin{gathered}
\beta\left(t_{n+1}\right)=\hat{U}^{n+1}-u_{h}\left(t_{n+1}\right), \\
\hat{U}^{n+1}=u_{h}\left(t_{n}\right)+\tau F\left(\frac{1}{2} u_{h}\left(t_{n}\right)+\frac{1}{2} \hat{U}^{n+1}\right) .
\end{gathered}
$$

$$
\beta\left(t_{n+1}\right)=d_{1}\left(t_{n+1}\right)+\tau F\left(\frac{1}{2} u_{h}\left(t_{n}\right)+\frac{1}{2} \hat{U}^{n+1}\right)-\tau F\left(\frac{1}{2} u_{h}\left(t_{n}\right)+\frac{1}{2} u_{h}\left(t_{n+1}\right)\right) .
$$

By applying the same techniques which are used for deriving the $C$-stability inequality (6.16) one can prove that

$$
\left\|\beta\left(t_{n+1}\right)\right\|_{2} \leq\left(1-\frac{1}{2} \tau \nu\right)^{-1}\left\|d_{1}\left(t_{n+1}\right)\right\|_{2}, \tau \nu<2,
$$

where $v$ is again an upper bound for the logarithmic norm (here computed on the line segment connecting $\hat{U}^{n+1}$ and $\left.u_{h}\left(t_{n+1}\right)\right)$.

Next we introduce a second defect

$$
d_{2}\left(t_{n+1}\right)=u_{h}\left(t_{n}\right)+\frac{1}{2} \tau F\left(u_{h}\left(t_{n}\right)\right)+\frac{1}{2} \tau F\left(u_{h}\left(t_{n+1}\right)\right)-u_{h}\left(t_{n+1}\right),
$$

which is associated to the trapezoidal rule (Crank-Nicholson), and establish that for the ODE system (6.5) the difference $d\left(t_{n+1}\right)=d_{1}\left(t_{n+1}\right)-d_{2}\left(t_{n+1}\right)$ is given by

$$
\begin{gathered}
d\left(t_{n+1}\right)=\frac{1}{2} \tau B\left(\frac{1}{2} u_{h}\left(t_{n}\right)+\frac{1}{2} u_{h}\left(t_{n+1}\right)\right)\left(u_{h}\left(t_{n}\right)+u_{h}\left(t_{n+1}\right)\right) \\
-\frac{1}{2} \tau B\left(u_{h}\left(t_{n}\right)\right) u_{h}\left(t_{n}\right)-\frac{1}{2} \tau B\left(u_{h}\left(t_{n+1}\right)\right) u_{h}\left(t_{n+1}\right) .
\end{gathered}
$$

It follows that $d$ is independent of the space differencing. A straightforward Taytor expansion shows that

$$
\left\|d\left(t_{n+1}\right)\right\|_{2} \leq c \tau^{3}, c \text { independent of } \tau \text { and } h .
$$

The final step in the proof of consistency is the examination of $d_{2}$. Using the space truncation error (3.1) we can write

$$
d_{2}\left(t_{n+1}\right)=u_{h}\left(t_{n}\right)-u_{h}\left(t_{n+1}\right)+\frac{1}{2} \tau\left(\dot{u}_{h}\left(t_{n}\right)+\dot{u}_{h}\left(t_{n+1}\right)\right)+\frac{1}{2} \tau\left(\alpha\left(t_{n}\right)+\alpha\left(t_{n+1}\right)\right) .
$$

From the second order consistency of the trapezoidal rule, the second order consistency of the discretization in space, the inequalities (6.20) and (6.23), we thus arrive at the consistency result 


$$
\left\|\tau^{-1} \beta\left(t_{n+1}\right)\right\|_{2} \leq C_{1} \tau^{2}+C_{2} h^{2}, \tau \nu<2,
$$

where $C_{1}$ and $C_{2}$ are constants independent of $\tau$ and $h$.

There remains to remove the boundedness hypothesis on the components of the fully discrete solutions $U^{n+1}$. This can be done in exactly the same manner as we did for the semi-discrete solution $U$. In conclusion, according to Theorem 4.1 we have proved that the fully discrete implicit midpoint solution $U^{n+1}$ converges in $l^{2}$ to the true PDE solution $u_{h}\left(t_{n+1}\right)$, as $\tau, h \rightarrow 0$, without any further condition on $\tau$ and $h$. Convergence of the fully discrete trapezoidal solution (Crank-Nicholson) can be proved in the same manner.

Remark 6.1: Substitution of $\chi=C_{1} \tau^{3}+C_{2} \tau h^{2}$ into (4.6) yields the corresponding bound for the discretization error $\varepsilon\left(t_{n+1}\right)$. Here $C_{0}>0$ as the amplification factor $k$ in (4.4) is given by $k=(2+\tau v) /(2-\tau v)$, where $v>0$ stands for an upper bound for the logarithmic matrix norm $\mu_{2}$ computed in a tube around $u_{h}(t), 0 \leq t \leq T$. Due to the earlier mentioned property of conservation we can deduce that in (4.6) the exponential behaviour for increasing time cannot be realistic. In fact, using conservation, we can state the crude bound

$$
\left\|\varepsilon\left(t_{n+1}\right)\right\|_{2} \leq\left\|U^{n+1}\right\|_{2}+\left\|u_{h}\left(t_{n+1}\right)\right\|_{2}=\left\|u_{h}(0)\right\|_{2}+\left\|u_{h}\left(t_{n+1}\right)\right\|_{2},
$$

which shows that $\varepsilon$ is bounded in time.

\section{Some Remarks on the Use of the Time Truncation Error}

To conclude this paper we consider briefly the possibility of a convergence analysis which is set up completely in accordance with the MOL approach. More precisely, an analysis with proves the convergence of the ODE solution to the true PDE solution as $\rho(h) \rightarrow 0$ and, separately, the (uniform in $h$ ) convergence of the fully discrete solution to that of the ODE as $\tau \rightarrow 0$. The convergence of the discretization in space can be proved along the lines of Section 3 . The convergence proof of the time integration then requires the use of the time truncation error $y$ given by $\left(4.1^{\prime}\right)$ in combination with the property of $C$-stability. Let $\xi$ be an upper bound for $\gamma$. Supposing $C$-stability and using $\gamma\left(t_{n+1}\right)$ instead of $\beta\left(t_{n+1}\right)$ in the derivations of Section 4 , one thus arrives at the error bound

$$
\left\|\varepsilon\left(t_{n+1}\right)\right\| \leq\left\|\eta\left(t_{n+1}\right)\right\|+\left\|\delta\left(t_{n+1}\right)\right\|,
$$

where, similar to (4.6), the time integration error $\delta\left(t_{n+1}\right)$ satisfies

$$
\left\|\delta\left(t_{n+1}\right)\right\| \leq \begin{cases}\tau^{-1} \xi\left|C_{0}\right|^{-1}, & C_{0}<0 \\ \tau^{-1} \xi t_{n+1}, & C_{0}=0, \\ \tau^{-1} \xi C_{0}^{-1}\left(e^{c_{0} t_{n+1}}-1\right), & C_{0}>0 .\end{cases}
$$

Obviously, the task is now to prove that $\tau^{-1} \xi \rightarrow 0$ as $\tau \rightarrow 0$, uniformly in the grid distance. More precisely, a constant $C_{3}$ and an integer $q$ should exist, both independent of $\tau$ and $h$, such that

$$
\left\|\gamma\left(t_{n+1}\right)\right\| \leq C_{3} \tau^{q}, \tau \rightarrow 0 .
$$


As pointed out earlier in [9] this hypothesis of time consistency uniform in $h$ may be difficult to verify. For example, consider the inequality (5.14). Using the present approach, one finds

$$
\left\|\gamma\left(t_{n+1}\right)\right\|_{\infty} \leq \frac{1}{1-\tau f_{1}} \frac{1}{2} \tau^{2} \max _{j}\left|\ddot{U}_{j}\left(t_{n}+\theta_{j} \tau\right)\right|, 0 \leq \theta_{j} \leq 1, \tau f_{1}<1 .
$$

In order to ensure time consistency uniform in $h$, it is necessary to prove that the second derivative $\ddot{U}(t)$ of the semi-discrete solution $U(t)$ is bounded as $\rho(h) \rightarrow 0$. Despite convergence of $U(t)$ to $u_{h}(t)$ this property of boundedness of $\ddot{U}(t)$ requires an additional investigation. This is the main reason why we prefer the direct approach for the consistency part in the convergence proof. An additional reason lies in the fact that the approach via (7.1) is not able to account for cancellations between errors in the time and space discretization (an example of such a cancellation is provided by the Douglas high accuracy scheme for the heat equation, [8], p. 190, formula G).

\section{References}

[1] Axelsson, O.: Error estimates over infinite intervals of some discretizations of evolution equations. Technical Report, Catholic University of Nijmegen, 1983 (to appear in BIT).

[2] Burrage, K., Butcher, J. C.: Stability criteria for implicit Runge-Kutta methods. SIAM J. Numer. Anal. 16, 46-57 (1979).

[3] Dahlquist, G.: Stability and error bounds in the numerical integration of ordinary differential equations. Trans. Royal Inst. Techn., No 130, Stockholm, 1959.

[4] Dekker, K., Verwer, J.G.: Stability of Runge-Kutta Methods for Stiff Nonlinear Differential Equations. Amsterdam-New York-Oxford: North-Holland 1984.

[5] Frank, R., Schneid, J., Ueberhuber, C. W.: The concept of B-convergence. SIAM J. Numer. Anal. $18,753-780(1981)$.

[6] Griffiths, D. F., Mitchell, A. R., Morris, J. LI: A numerical study of the nonlinear Schrödinger equations. Comp. Meth. Appl. Mech. Engn. 45, 177 - 215 (1984).

[7] Kreiss, H. O.: Über die Stabilitätsdefinition für Differenzengleichungen die partielle Differentialgleichungen approximieren. BIT 2, 153-181 (1962).

[8] Richtmyer, R. D., Morton, K. W.: Difference Methods for Initial Vale Problems. New York: Interscience. 1967.

[9] Sanz-Serna, J. M.: Convergent approximations to PDEs and contractivity of methods for stiff systems of ODEs. In: Actas del VI CEDYA, Jaca, 1983, pp. 488-493 (this paper is a highly condensed version of a report which is available on request).

[10] Sanz-Serna, J. M.: Methods for the numerical solution of the nonlinear Schrödinger equation. Math. of Comput. 43, $21-24$ (1984).

[11] Verwer, J. G., Dekker, K.: Step-by-step stability in the numerical solution of partial differential equations. Report NW 161/83, Mathematical Centre, Amsterdam, 1983.

[12] Verwer, J. G.: Contractivity of locally one-dimensional splitting methods. Numer. Math. 44, $247-259$ (1984).

[13] Wirz, H. J.: On iterative solution methods for systems of partial differential equations. Lecture Notes in Mathematics, Vol.679, pp. 151-163. Berlin-Heidelberg-New York: Springer 1978.

J. G. Verwer

Centre for Mathematics and Computer Science

Foundation Mathematical Centre

Kruislaan 413

1098 SJ Amsterdam

The Netherlands

\section{J. M. Sanz-Serna}

Universidad de Valladolid

Facultad de Ciencias

Dpto. de Ecuaciones Funcionales

Valladolid

Spain 